Supplemental Materials for

\title{
A one-dimensional seismic model for Uturuncu volcano, Bolivia, and its impact on full moment tensor inversions
}

Weisen Shen ${ }^{1}$, Celso Alvizuri ${ }^{2}$, Fan-Chi Lin ${ }^{3}$, Carl Tape ${ }^{2}$

1. Department of Earth and Planetary Sciences, Washington University in St Louis, St Louis, MO, 63130, USA

2. Geophysical Institute and Department of Geosciences, University of Alaska, Fairbanks, USA

3. Department of Geology and Geophysics, University of Utah, 271 Frederick Albert Sutton Building, Salt Lake City, UT 84112, USA

\section{Figure Captions}

Figure S1: Waveform fits for event B (event ID 20100516085611725), using the 1D velocity model. See caption of Figure 5 for explanation. The minimum misfit moment tensor here has magnitude Mw3.0 and VR = 26.0\%.

Figure S2: Waveform fits for event A (event ID 20100516063454464), using a homogeneous halfspace. See caption of Figure 5 for explanation. The minimum misfit moment tensor here has magnitude Mw2.9 and VR = 25.2\%.

Figure S3: Full moment tensor misfit summary for event A (event ID 20100516063454464). The waveform fits are shown in Figure 4. The caption below (including equation references) is directly from Figure 6 of Alvizuri and Tape (2016). (a) Map of source location (red star) and stations used in the inversion for this event. The station is colored blue if the observed firstmotion polarity on the vertical component is up (compression) and white if it is down (dilatation). (b) Contour plot of the polarity misfit on the lune. Not to be confused with the waveform misfit, the polarity misfit $\mathrm{n}(\mathrm{M})$ (Eq. 5) for a moment tensor $\mathrm{M}$ is the number of stations where the observed first-motion polarity differs from the polarity predicted from $\mathrm{M}$. The polarity misfit $n(\Lambda)$ (Eq. 6) at a point $\Lambda$ on the lune is then the minimum of $n(M)$ for moment tensors $M$ having source type $\Lambda$. At each point $\Lambda$ in the region where $n(\Lambda)=0$ (blue), there is therefore a moment tensor $\mathrm{M}$ with source type $\Lambda$ that has correct polarities, that is, polarities that match the observed polarities at all 11 stations. (c) Contour plot of the variance reduction $\operatorname{VR}(\Lambda)$. The variance reduction $\operatorname{VR}(\Lambda)$ at a point $\Lambda$ is the maximum variance reduction $\operatorname{VR}(\mathrm{M})$ for moment tensors $\mathrm{M}$ that have source type $\Lambda$ and that have correct polarities. (Compare Eq. 7, which is the analog of $\operatorname{VR}(\Lambda)$ for misfit.) Large values (blue) of VR represent better fit between observed and synthetic waveforms. Since $\mathrm{M}$ is required to have correct polarities, the plot of $\operatorname{VR}(\Lambda)$ is only defined on the region $n(\Lambda)=0$. The beachball plotted at each point $\Lambda$ of this region is the moment tensor $\mathrm{M}(\Lambda)$ that maximizes $\operatorname{VR}(\mathrm{M})$ with _ fixed. Of the beachballs $\mathrm{M}(\Lambda)$, our desired solution M0 (green box) is the one with largest VR. The gray arcs on the lune are the great circle arcs $\lambda 1=0, \lambda 2=0$, and $\lambda 3=0$ (white, green, and red in Figure 1 ). Selected eigenvalue triples (black dots) on the boundary of the lune are indicated, with the understanding that the triples need to be normalized. The positive isotropic source $(1,1,1)$ is at the top, the negative isotropic source $(-1,-1,-1)$ is at the bottom, and the double couple $(1,0,-1)$, not shown, 
would be at the center of the lune. (d) The moment tensor M0, the same as in (c) but plotted in a lower-hemisphere projection. For this event, all ray paths travel upward from the source to the stations, so the stations to the east, having upward (compressional) first motions, are plotted on the left of the beachball at the antipode of the ray path direction.

Figure S4: Misfit summary for event A (event ID 20100516063454464), using a homogeneous model. See caption of Figure S3 for explanation. The waveform fits are shown in Figure 6.

Figure S5: Misfit summary for event B (event ID 20100516085611725), using a 1D velocity model. See caption of Figure S3 for explanation. The waveform fits are shown in Figure S1.

Figure S6: Misfit summary for event B (event ID 20100516085611725), using a homogeneous model. See caption of Figure S3 for explanation. The waveform fits are shown in Figure S2. 


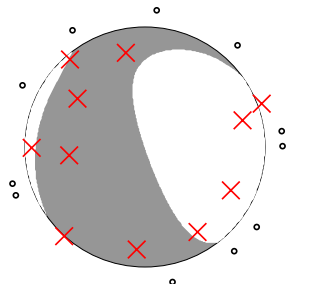

Event 20100516085611725 Model and Depth utu1D_010

FM 3378873 Mw $3.00 \gamma 9 \delta$ o rms 9.656e-08 VR 26.0

Filter periods (seconds): Body:0.10-0.50. Surf:2.00-4.00

\# norm L1 \# Pwin 1.5 Swin 60 \# N 11 Np 0 Ns 33
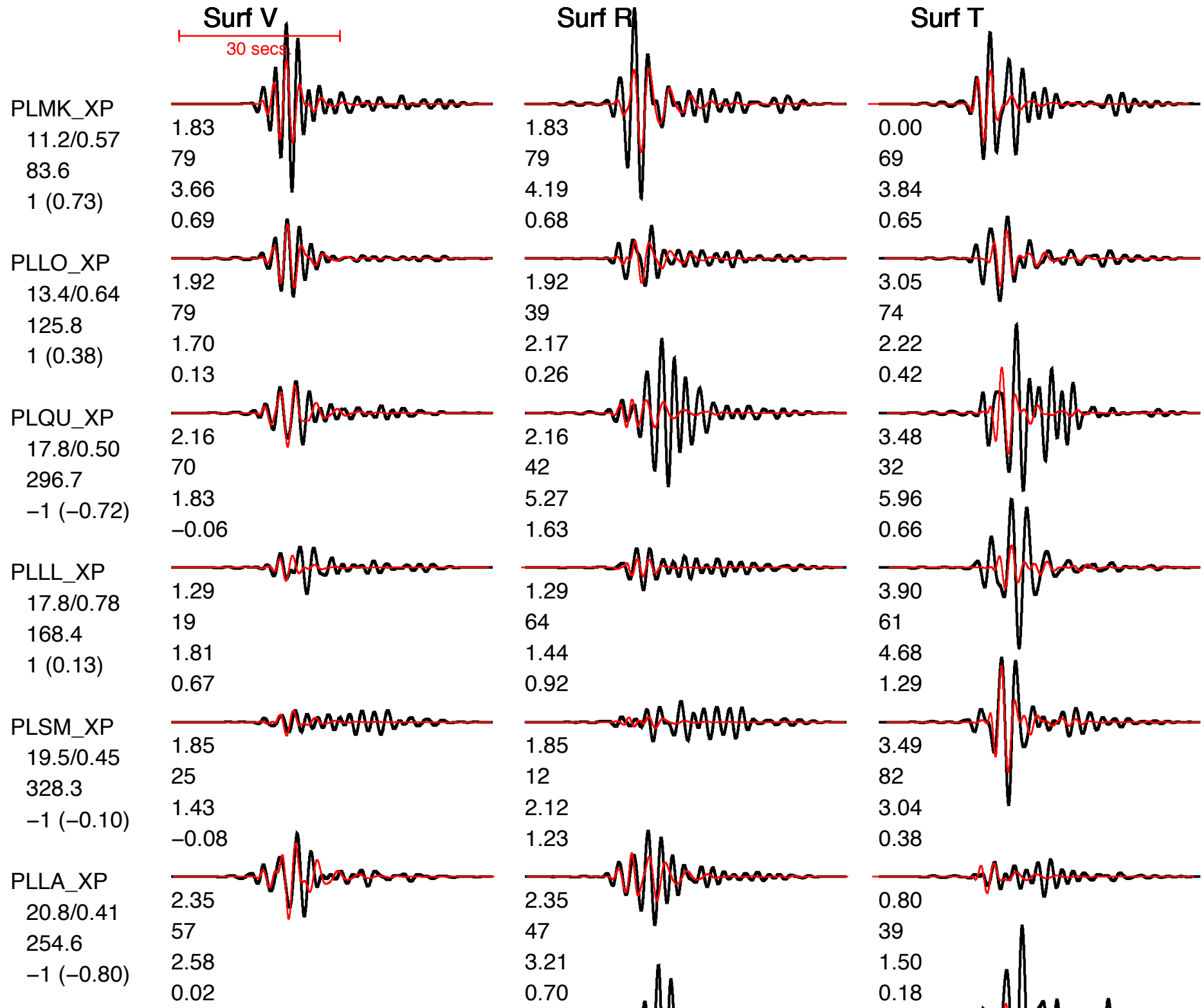

3.84
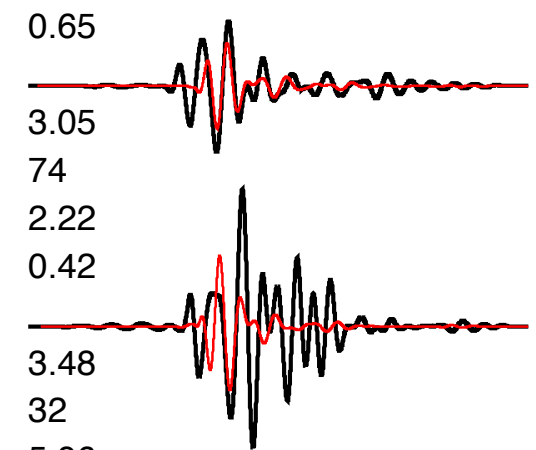

5.27

1.63
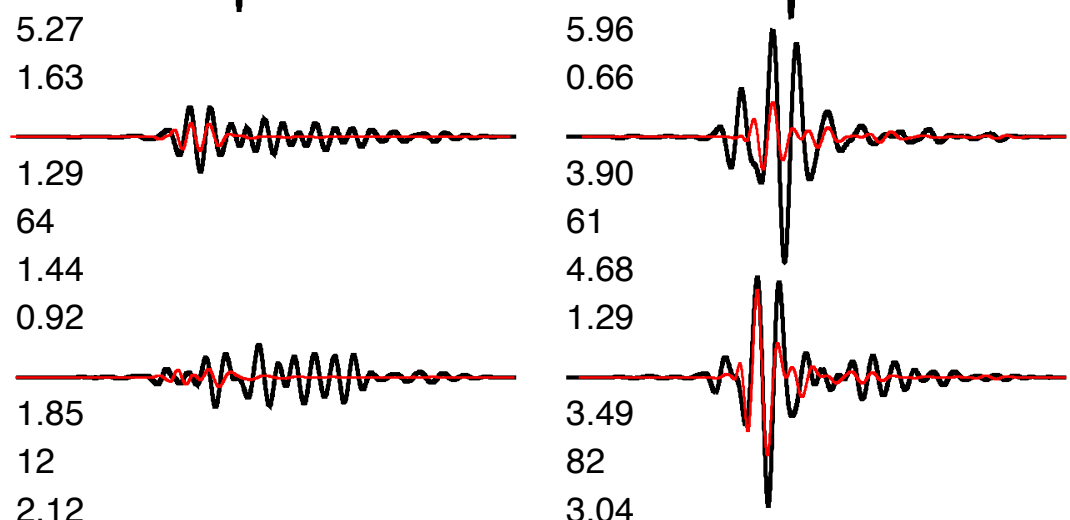

64

1.44

0.92

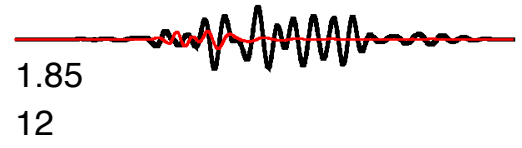

2.12

3.04

1.23

0.38
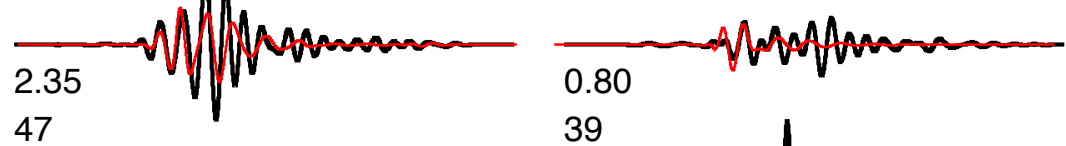

47

39

3.21
0.70

PLTM_XP

$22.2 / 0.75$

4.8

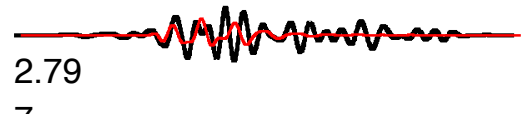

0.70 7

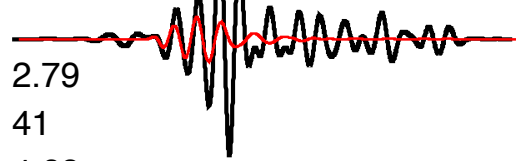

$1(0.52)$

PLRR_XP

$31.2 / 0.74$

89.7

1.79

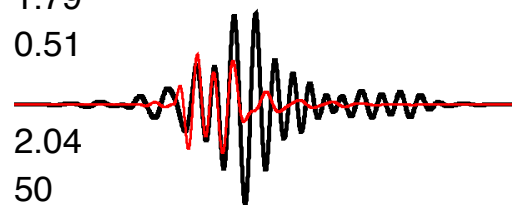

4.68

1.65

$1(0.01)$

PLAR_XP

$32.7 / 0.64$

139.4

$1(0.06)$

4.32

0.69

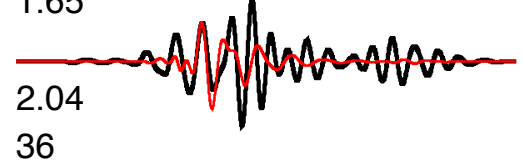

1.50

0.18

4.0

36

7.43

1.36

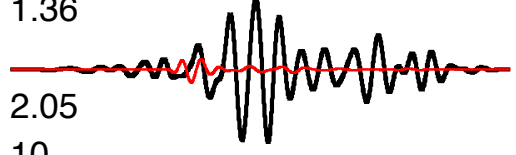

10

4.31

3.26

0.31
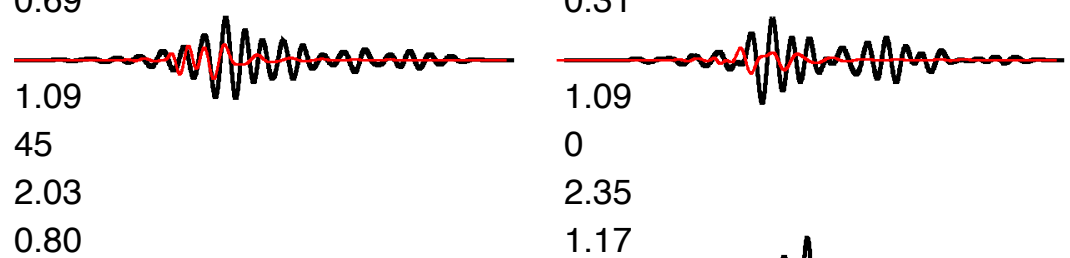

1.72

1.37

68

PL03_XP

36.9/0.51

42.1

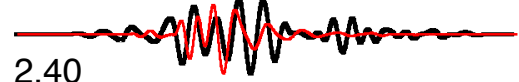

1.17

1.08
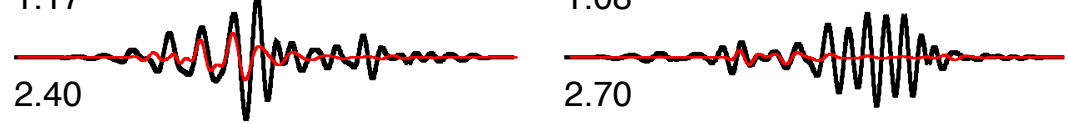
26

67

26

$1(0.27)$

PLSQ_XP

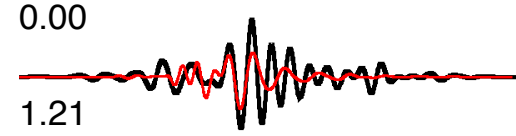

0.96

1.88 


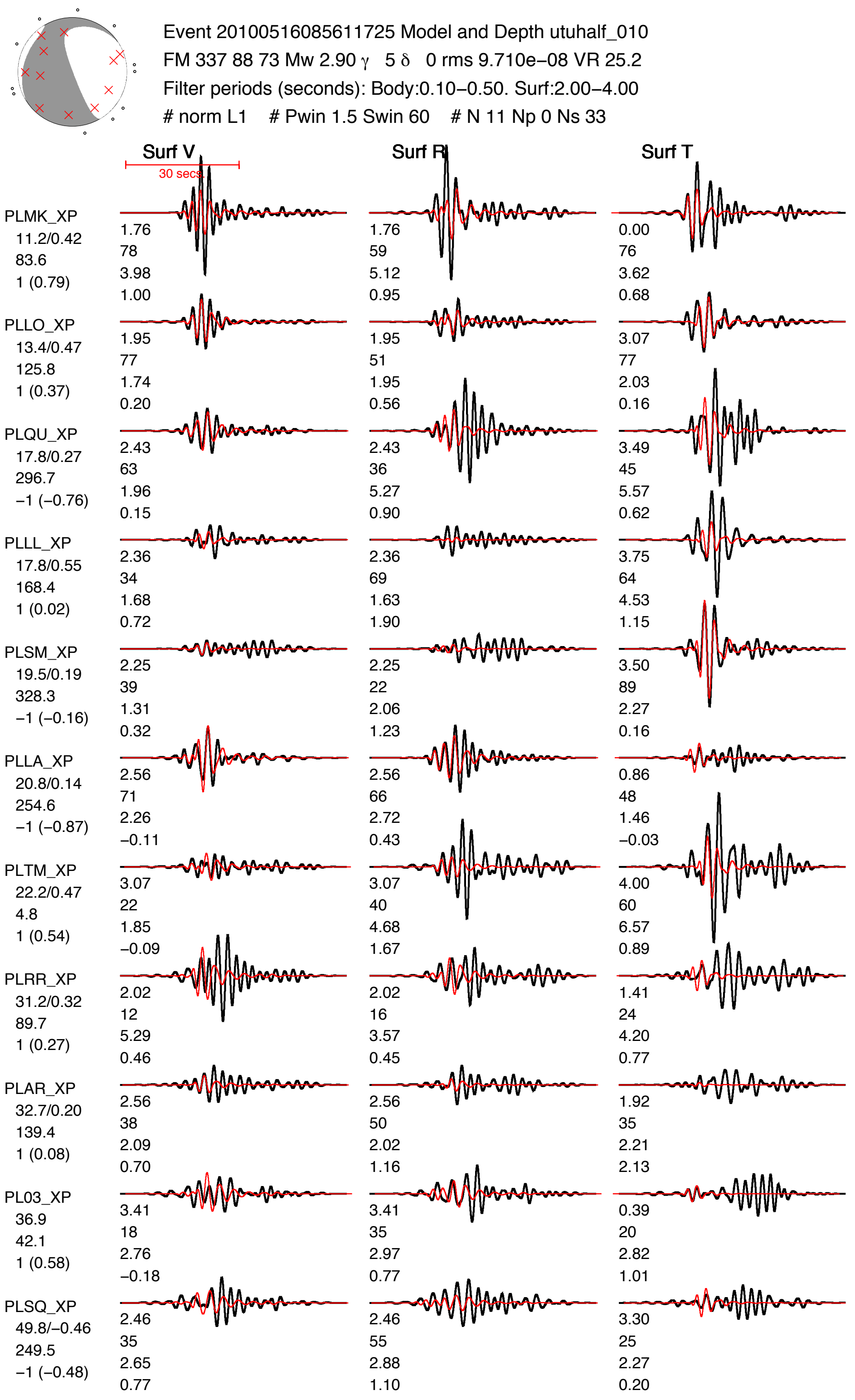


Event 20100516063454464, M 2.90 Lon -67.1856, Lat -22.2600 Dep 0.6 km (inversion 4 km)

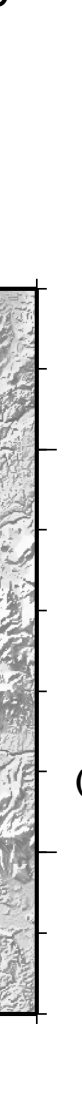

(b)

(a) $\quad$ Up: 7 O Down: 4
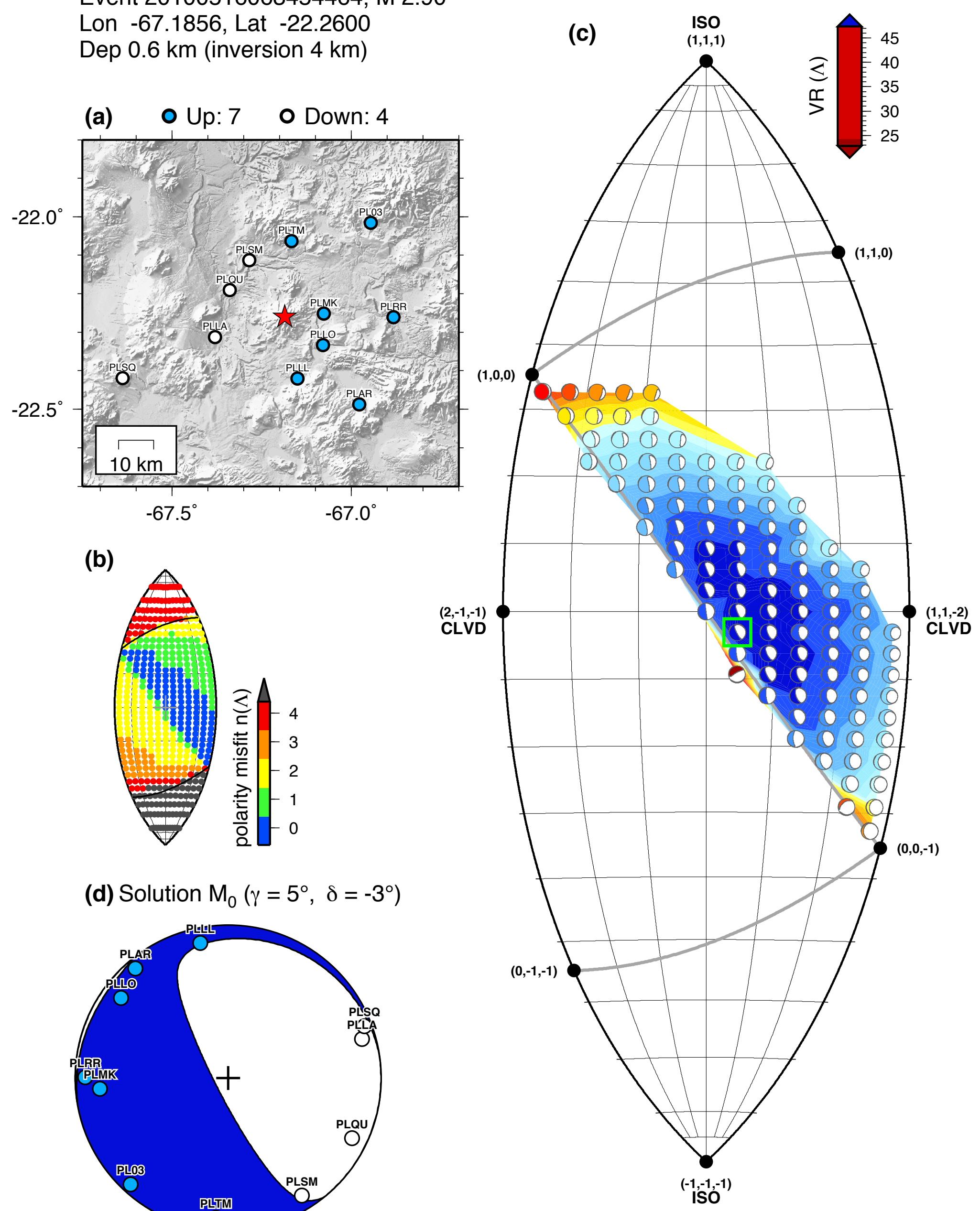

(d) Solution $M_{0}\left(\gamma=5^{\circ}, \delta=-3^{\circ}\right)$

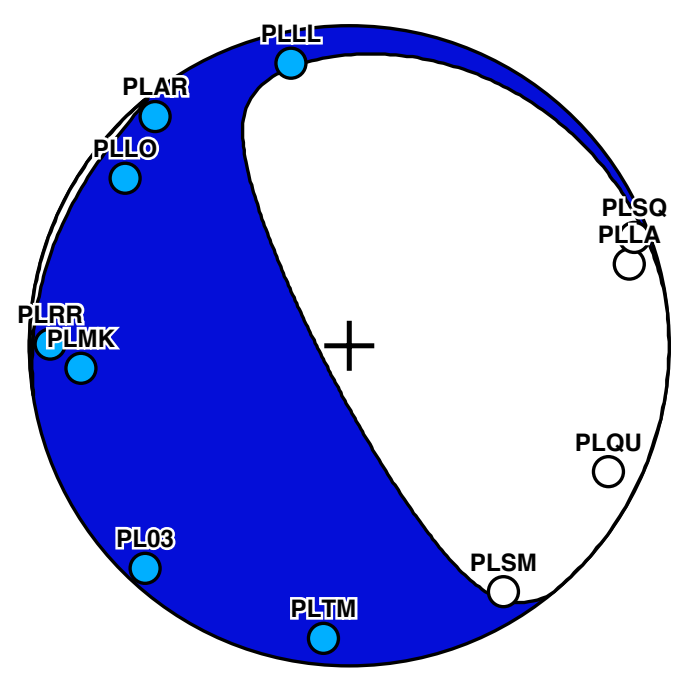

iso 
Shen, Celso, Lin, and Tape, Fig. S4

Event 20100516063454464, M 2.80 Lon -67.1856, Lat -22.2600 Dep 0.6 km (inversion 4 km)
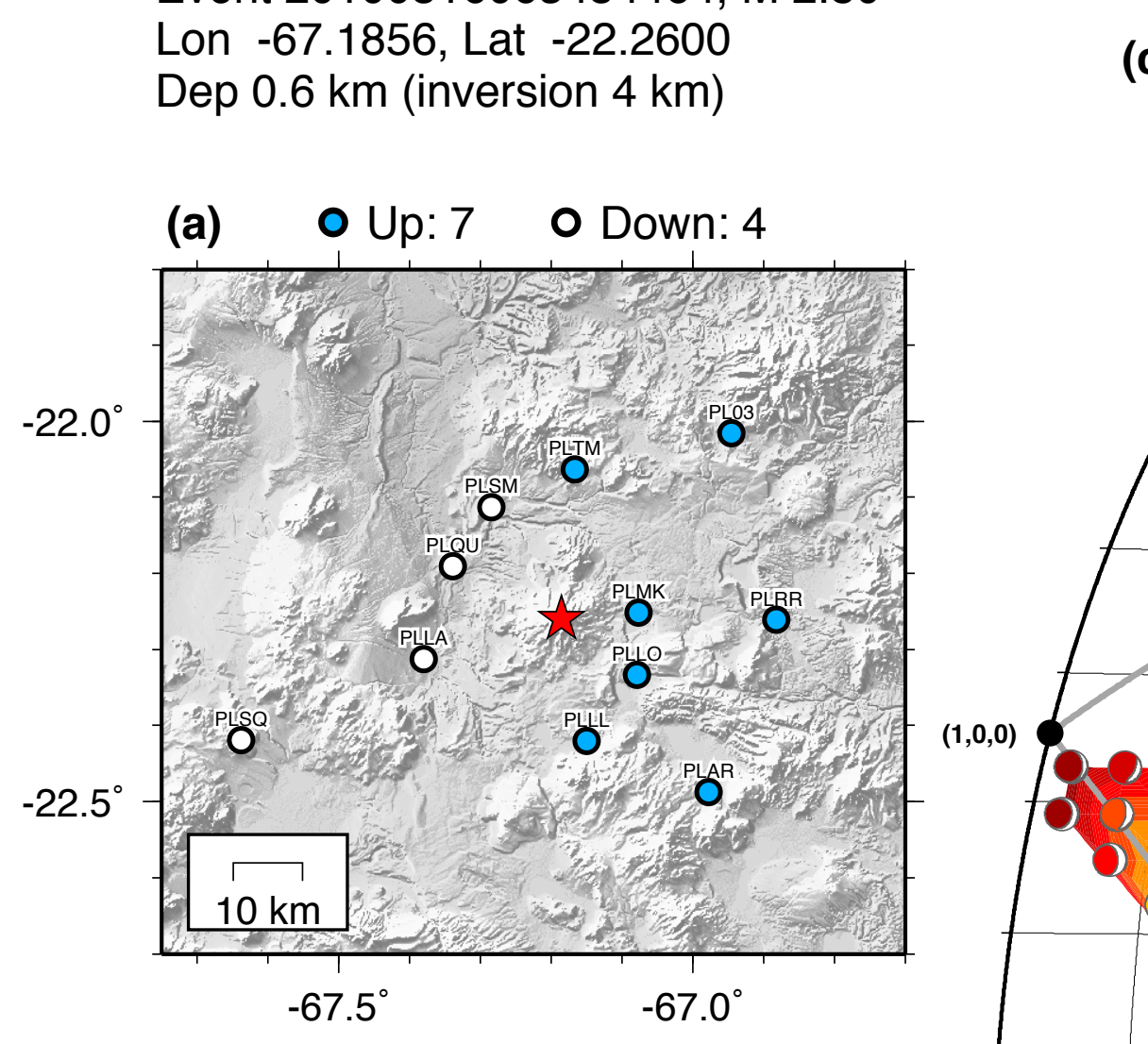

(a) O Up: 7 O Down: 4

(b)

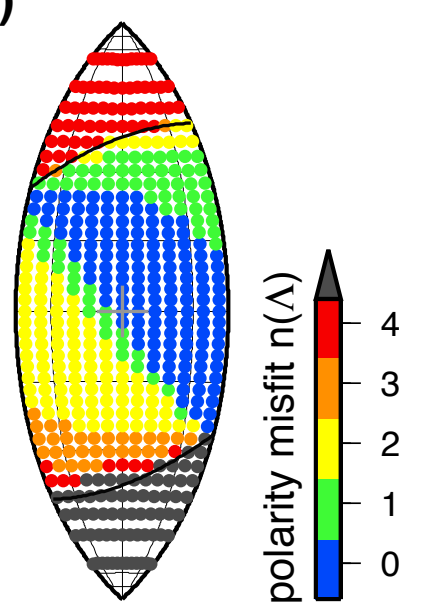

(d) Solution $\mathrm{M}_{0}\left(\gamma=18^{\circ}, \delta=6^{\circ}\right)$

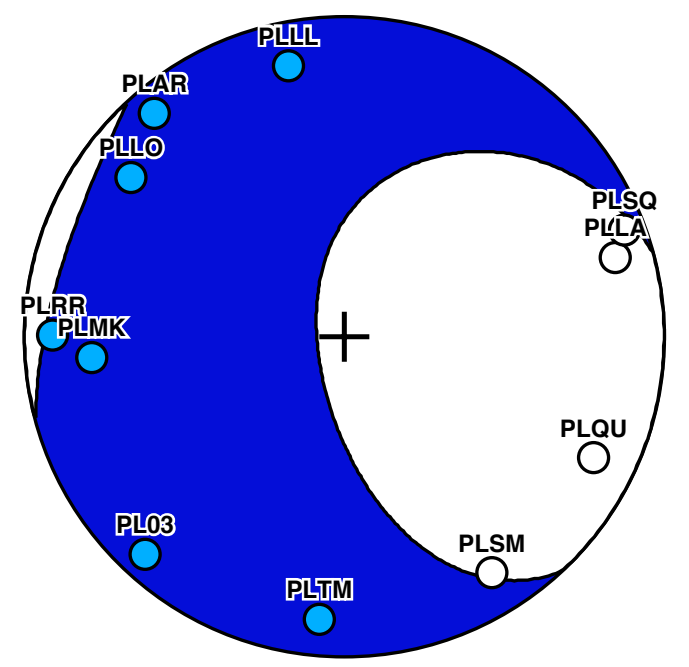

$(2,-1,-1)$ CLVD

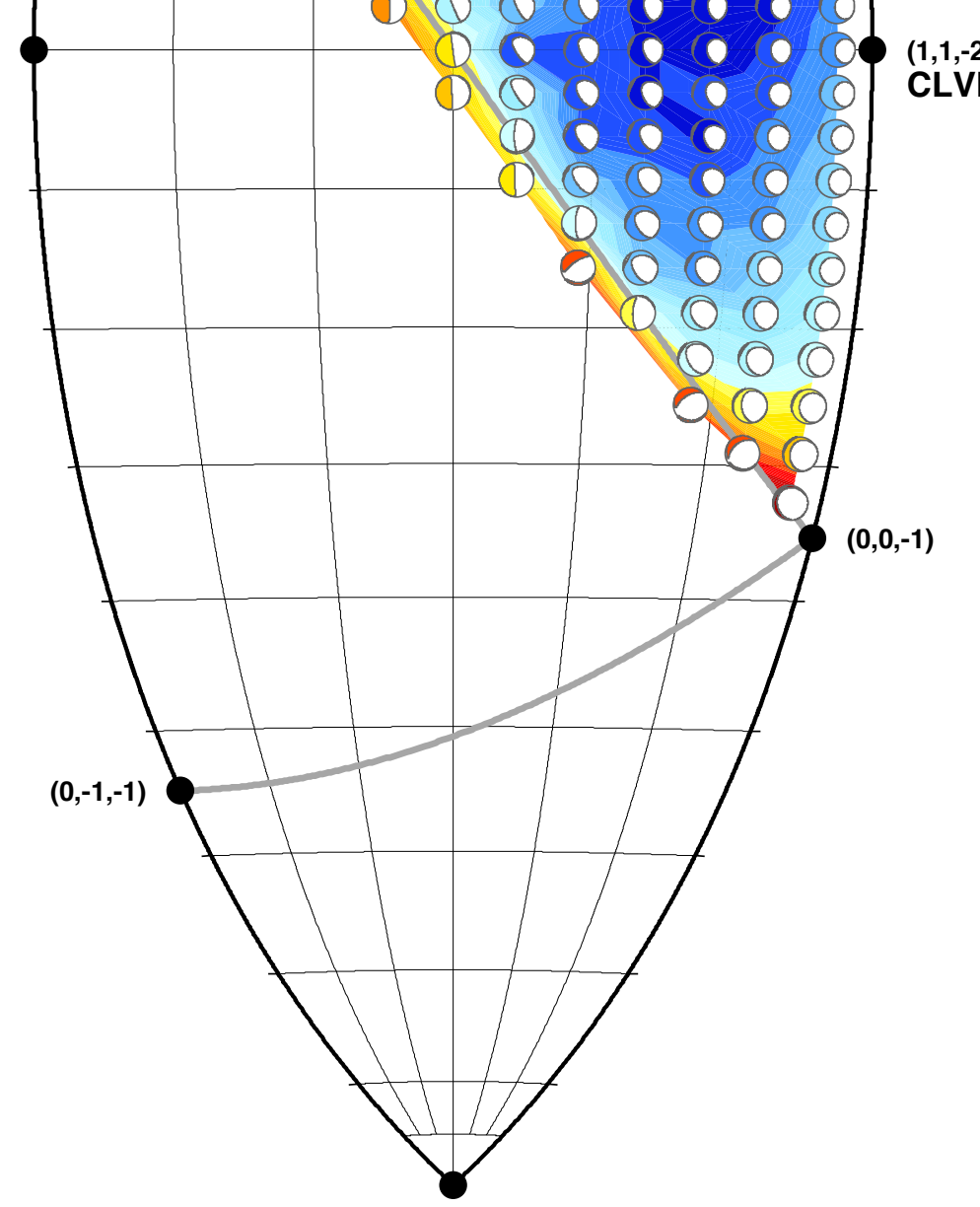

(-1,-1,-1)
$(1,1,-2)$
CLVD

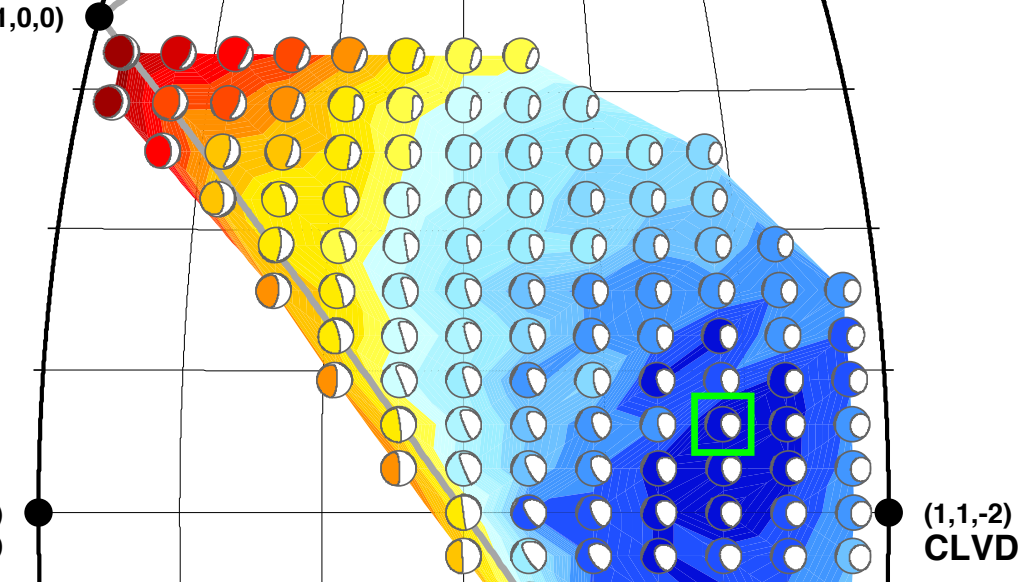


Shen, Celso, Lin, and Tape, Fig. S5

Event 20100516085611725, M 3.00 Lon -67.1849, Lat -22.2629

Dep 5.1 km (inversion $10 \mathrm{~km}$ )

(a) $\quad$ Up: 7 O Down: 4

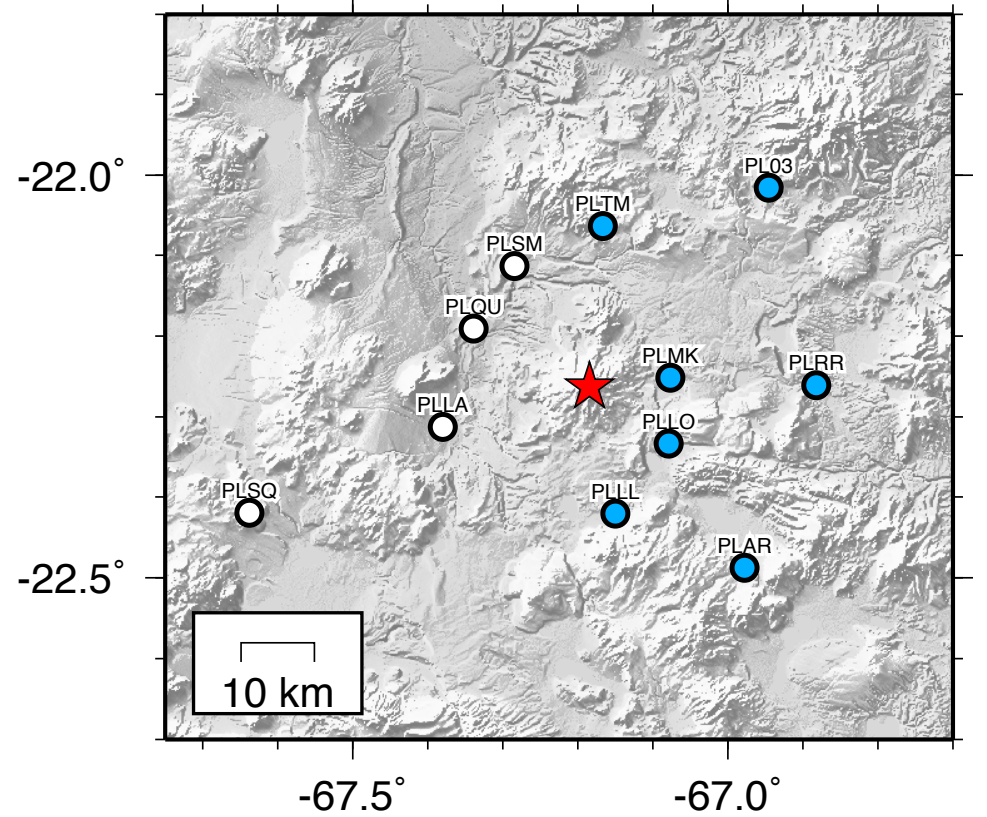

(b)

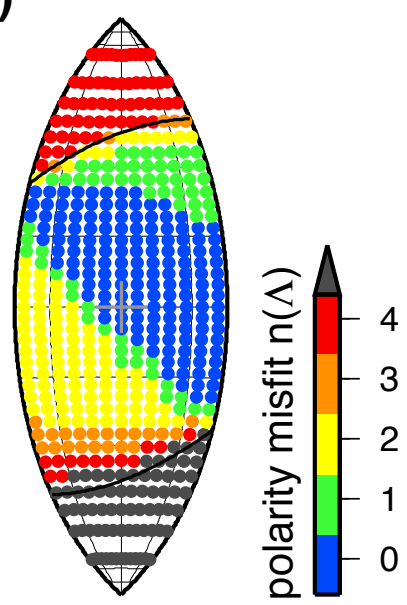

(d) Solution $M_{0}\left(\gamma=9^{\circ}, \delta=-0^{\circ}\right)$

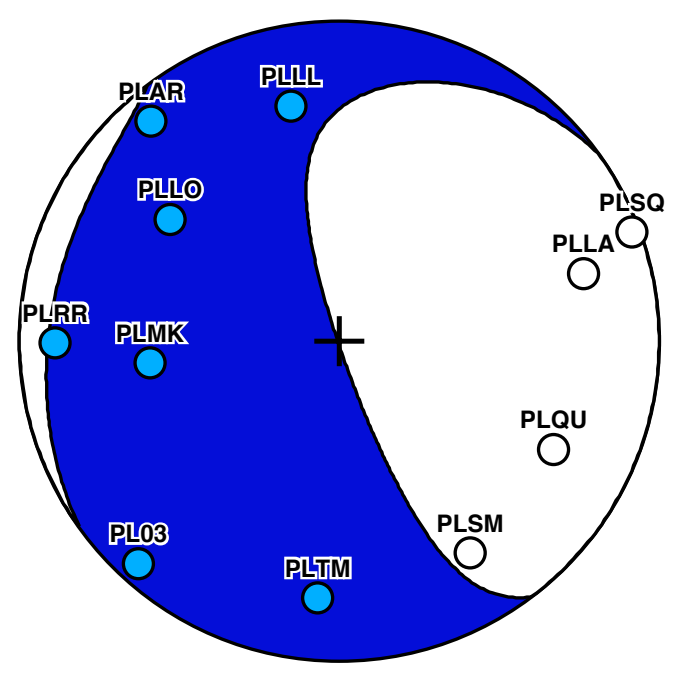

(c)

$(1,0,0)$

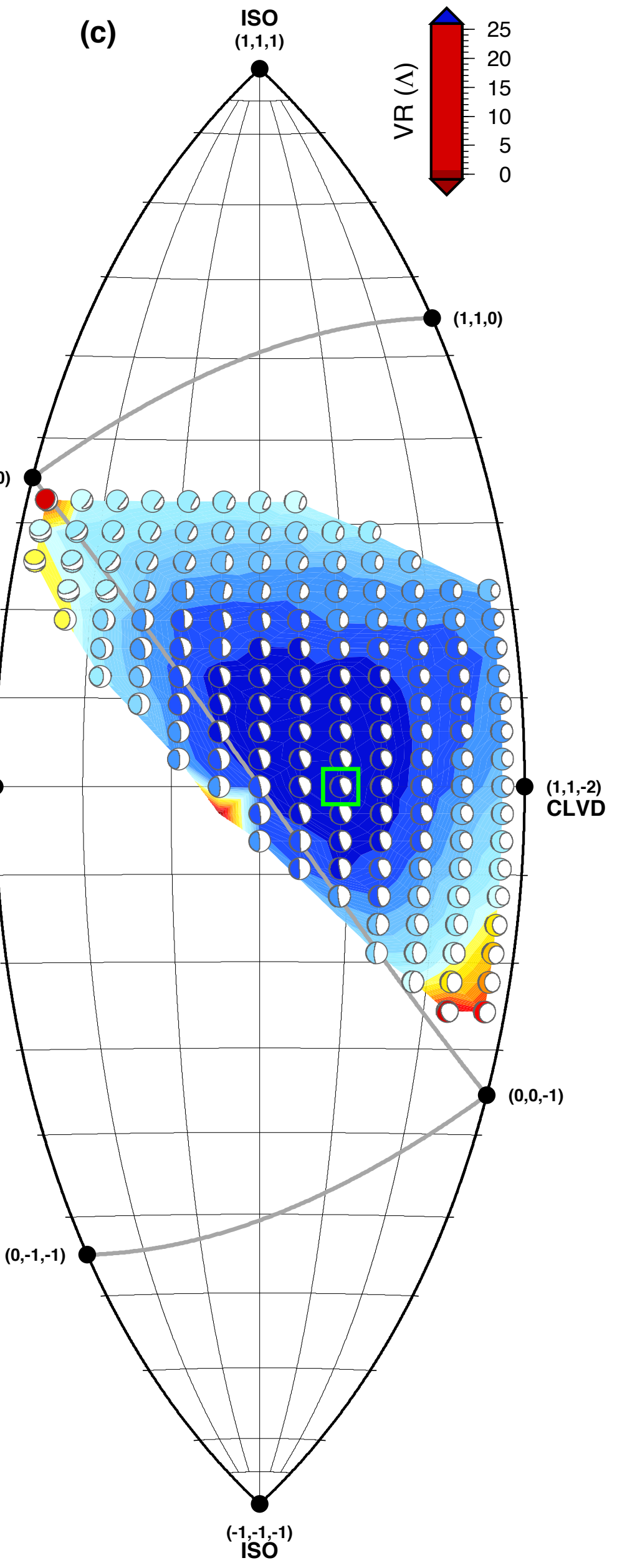


Shen, Celso, Lin, and Tape, Fig. S6

Event 20100516085611725, M 2.90 Lon -67.1849, Lat -22.2629

Dep 5.1 km (inversion 10 km)

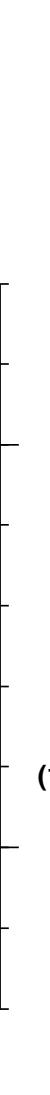

(b)

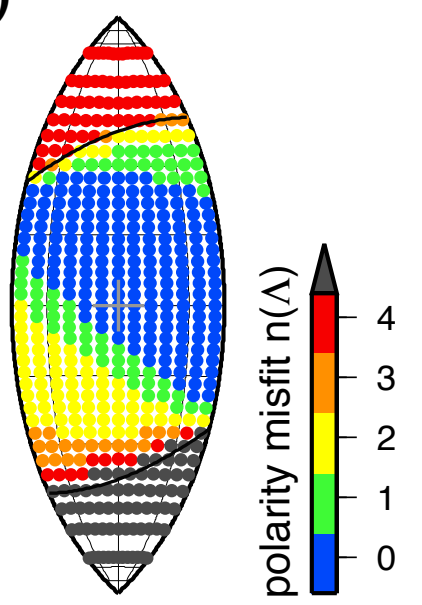

(d) Solution $\mathrm{M}_{0}\left(\gamma=5^{\circ}, \delta=-0^{\circ}\right)$

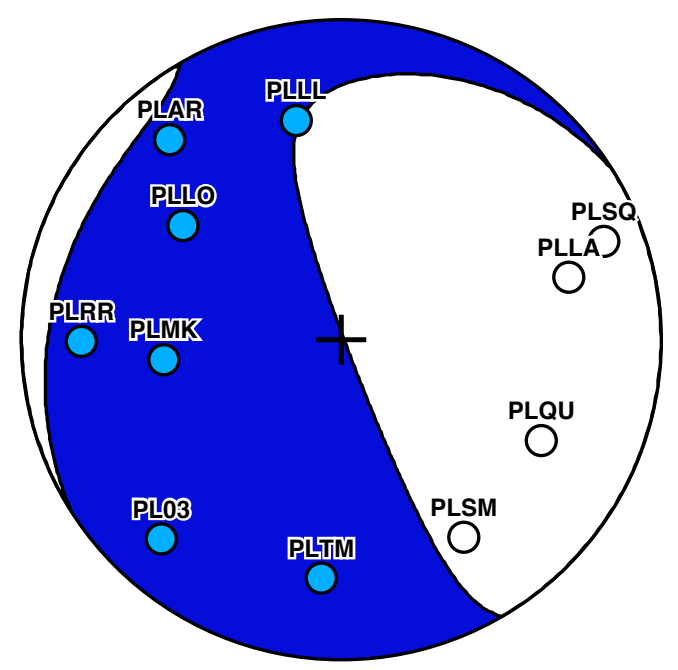

$(2,-1,-1)$

(c)

$(1,1,1)$

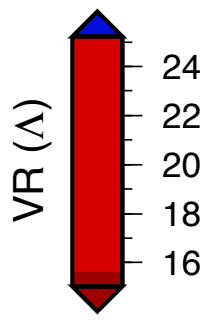

$(1,0,0)$

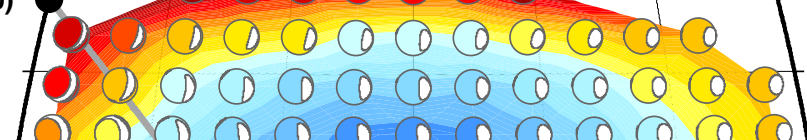

10000000000

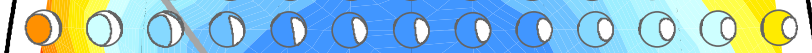

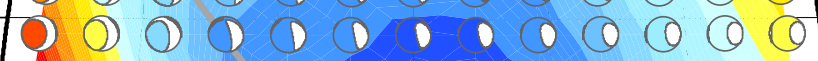

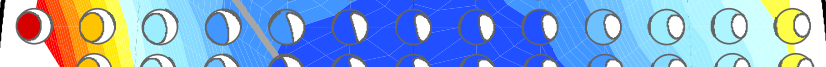

2) $00 \begin{array}{llllllllll}0 & 1 & 1 & 1 & 0 & 0 & 0 & 0 & 0\end{array}$

(2) 00 - 0.00000

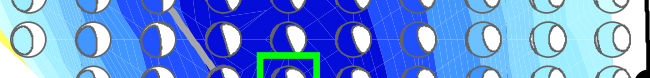
CLVD

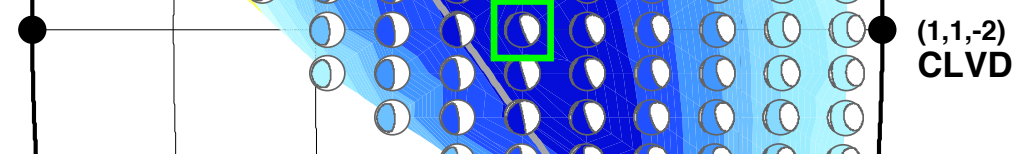

$(1,1,-2)$

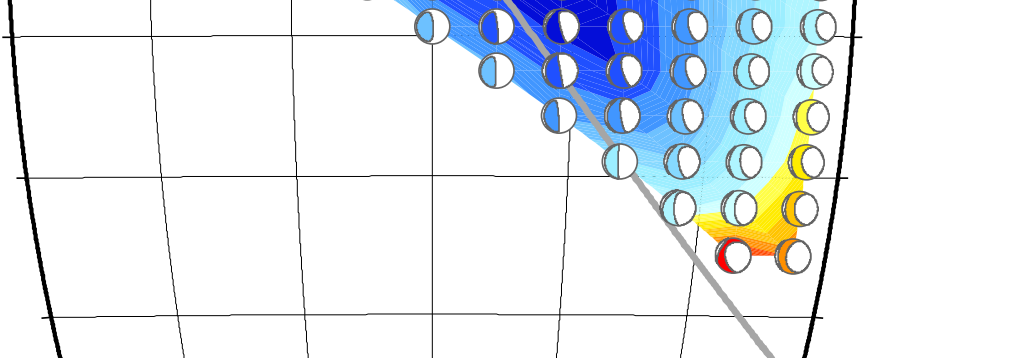

$(0,-1,-1)$

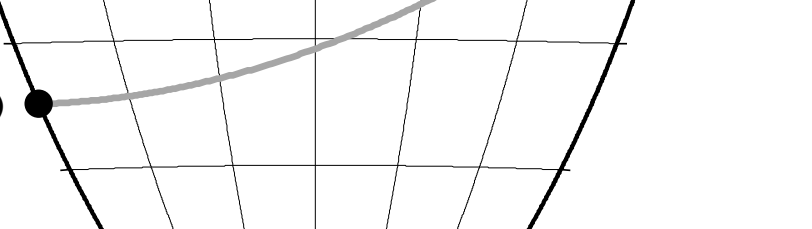

